\title{
A Cardiologia na Pandemia COVID-19: Que Impacto na Atividade Clínica?
}

\section{Cardiology in the Pandemic COVID-19: What is the Impact in Clinical Activity?}

Mário Oliveira, Paulino Sousa², Lídia Sousa³, Cláudia Mendes², Marta Labucha², Filipe Macedo²

\section{RESUMO}

A pandemia COVID-19 implicou alterações no funcionamento do sistema de saúde, com interrupção da atividade clínica programada, que, associada ao receio pelos doentes do risco de contaminação, condicionou o atendimento médico. No presente artigo analisámos os dados da atividade nos centros de Cardiologia dos hospitais CUF Infante Santo e CUF Porto durante o estado de emergência nacional. Estes hospitais, com características semelhantes na multifuncionalidade cardiovascular e integrados nos centros para tratamento da COVID-19, tiveram uma redução superior a 70\% nas consultas presenciais, ultrapassando os 60\% para a quase totalidade dos exames complementares de diagnóstico, em comparação com o período homólogo de 2019. Estes valores foram mais acentuados na evolução do primeiro quadrimestre de 2020, sobretudo à custa de atividade residual em abril. Os procedimentos invasivos eletivos também diminuíram, apresentando uma queda mais marcada em abril. No entanto, as intervenções urgentes por síndroma coronária aguda mantiveram-se sem variação face a igual período de 2019. Reforça-se a necessidade de divulgar a importância de recorrer às urgências hospitalares e da manutenção das normas de segurança na retoma gradual da atividade, e aborda-se o papel crescente da telemedicina e assistência domiciliária, bem como da reorganização do funcionamento das equipas hospitalares para melhorar a dinâmica multidisciplinar nas várias componentes da Cardiologia.

PALAVRAS-CHAVE: COVID-19; Doenças Cardiovasculares; Hospitais

1. Centro do Coração, Hospital CUF Infante Santo e Laboratório de Eletrofisiologia, Hospital CUF Porto, Porto, Portugal. 2. Centro do Coração, Hospital CUF Porto, Porto, Portugal. 3.Centro do Coração, Hospital CUF Infante Santo, Lisboa, Portugal. 


\section{ABSTRACT}

The COVID-19 Pandemic involved changes in the organization of the health care system, with interruption of the clinical programmed activity during the lockdown period, which, associated with the fear of contamination, had important repercussions in medical care. In this article, we analyzed data from the cardiology centers of CUF Infante Santo and CUF Porto hospitals during the state of national emergency. These hospitals, with similar characteristics in cardiovascular multifunctionality and integrated in the centers for treatment of COVID-19, had a reduction of more than $70 \%$ of face-to-face consultations, surpassing 60\% for almost all diagnostic tests, compared to the same period of 2019. This was even more pronounced when looking for the evolution during the first four months of 2020. Elective invasive procedures were also reduced, with a marked fall in April. However, urgent interventions due to acute coronary syndromes remained unchanged compared to the same period in 2019. The need to inform the population regarding the importance of using hospital emergencies and the maintenance of safety recommendations is reinforced, and the growing role of telemedicine and home-based care is addressed, as well as the reorganization of the hospital teams to improve the multidisciplinary dynamicity in Cardiology.

KEYWORDS: Cardiovascular Diseases; COVID-19; Hospitals

A atual pandemia causada pelo vírus SARS-CoV-2 veio impor a introdução de profundas alterações na organização e no funcionamento do sistema de saúde, nomeadamente com a súbita interrupção da atividade clínica programada dos diferentes departamentos, criando circuitos de atendimento para doentes infetados (ou com suspeita de infeção) e para doentes com teste negativo para COVID-19, e reorganizando as enfermarias, os serviços de urgência e unidades de cuidados intensivos, no que se refere à redistribuição de recursos humanos e condições logísticas. A aplicação dum modelo focado na segurança de doentes e profissionais de saúde, dotado de equipamentos de proteção individual adequados e de condições funcionais dinâmicas, capazes de responder a um surto que se estimou ser de grande expansão, implicou transformações abruptas em hospitais públicos e privados. Estas medidas associaram-se a regras de contenção da propagação da infeção, que incluíram o distanciamento social e confinamento, a desinfeção regular das mãos e superfícies de contacto, mas também o cancelamento da atividade eletiva nos cuidados de saúde. Este último, associado ao facto de muitos utentes evitarem recorrer às urgências hospitalares por receio de contaminação (com registo de reduções superiores a 50\% no número de admissões), condicionou o atendimento médico em geral, com expectável repercussão na Cardiologia.

Na realidade, a divulgação de que a população com fatores de risco e patologias cardiovasculares, e outras comorbilidades crónicas, representa um grupo particularmente vulnerável no contexto da COVID-19, sobretudo quando idosos, tem sido considerado como um dos aspetos que, somado aos anteriores, teve maior impacto na diminuição temporária dos cuidados de saúde no âmbito das doenças cardiovasculares.

Os Hospitais CUF Infante Santo (HCIS) e CUF Porto (HCP), com características semelhantes no que se refere a multifuncionalidades no diagnóstico e tratamento das doenças do foro da Cardiologia, envolvendo equipas em disponibilidade permanente no âmbito da via verde coronária e cuidados intensivos, com acesso às diferentes técnicas invasivas e não invasivas desta especialidade, foram integrados durante o período da pandemia COVID-19 no grupo de centros com internamento para doentes infetados pelo vírus SARS-CoV-2. Neste contexto, a atividade programada foi transitoriamente cancelada, com impacto no número de consultas e procedimentos efetuados. Nesta fase, em que se processa a retoma gradual do atendimento eletivo, analisámos a atividade clínica dos centros de Cardiologia destes hospitais durante o período de estado de emergência nacional.

Foram considerados: o número de consultas presenciais, visitas de cardiologista a doentes admitidos (atendimento permanente, internamento, unidade de cuidados intensivos), número de teleconsultas (modalidade iniciada em abril), eletrocardiogramas (ECG), provas de esforço, ecocardiogramas, registo de Holter, monitorização ambulatória da pressão arterial (MAPA) e procedimentos no laboratório de angiografia (coronariografias, angioplastias, dispositivos eletrónicos cardíacos implantáveis, ablações).

As Tabelas 1 e 2 mostram a comparação dos meses de março e abril de 2020 com o período homólogo do ano anterior, no que se refere ao número de consultas e exa- 
TABELA 1. Número de consultas e exames não invasivos de cardiologia para o hospital CUF Infante Santo (HCIS) - comparação dos meses de março e abril de 2020 com o período homólogo do ano anterior.

\begin{tabular}{|c|c|c|c|c|c|c|c|c|}
\hline CARDIOLOGIA HCIS & $\begin{array}{c}\text { março } \\
2020\end{array}$ & $\begin{array}{l}\text { abril } \\
2020\end{array}$ & $\begin{array}{c}\text { março/abril } \\
2020\end{array}$ & $\begin{array}{c}\text { março } \\
2019\end{array}$ & $\begin{array}{l}\text { abril } \\
2019\end{array}$ & $\begin{array}{c}\text { março/abril } \\
2019\end{array}$ & var abs & $\operatorname{var} \%$ \\
\hline $\mathrm{N}^{\circ}$ Consultas Presenciais & 337 & 48 & 385 & 749 & 645 & 1394 & -1009 & $-72 \%$ \\
\hline $\mathrm{N}^{\circ}$ Teleconsultas & - & 79 & 79 & - & - & - & - & - \\
\hline Visitas de Especialista (AP, INT, UCIP) & 14 & 3 & 17 & 9 & 10 & 19 & -2 & $-12 \%$ \\
\hline$N^{\circ} E C G$ & 651 & 320 & 971 & 1323 & 1220 & 2543 & -1572 & $-62 \%$ \\
\hline $\mathrm{N}^{\circ} \mathrm{ECO}$ & 337 & 48 & 385 & 632 & 662 & 1294 & -909 & $-70 \%$ \\
\hline $\mathrm{N}^{\circ}$ Holter & 59 & 6 & 65 & 131 & 114 & 245 & -180 & $-64 \%$ \\
\hline$N^{\circ} \mathrm{MAPA}$ & 30 & 0 & 30 & 73 & 54 & 127 & -97 & $-76 \%$ \\
\hline$N^{\circ} P E$ & 103 & 0 & 103 & 224 & 210 & 434 & -331 & $-76 \%$ \\
\hline
\end{tabular}

AP=atendimento permanente; INT=internamento; UCIP=unidade de cuidados intensivos polivalente; ECG=eletrocardiogramas; ECO=ecocardiogramas; Holter=registos de Holter; MAPA=monitorização ambulatória da pressão arterial; PE=provas de esforço; var abs=variação absoluta; var \%=percentagem de variação.

TABELA 2. Número de consultas e exames não invasivos de cardiologia para o hospital CUF Porto (HCP) - comparação dos meses de março e abril de 2020 com o período homólogo do ano anterior.

\begin{tabular}{|c|c|c|c|c|c|c|c|c|}
\hline CARDIOLOGIA HCP & $\begin{array}{c}\text { março } \\
2020\end{array}$ & $\begin{array}{l}\text { abril } \\
2020\end{array}$ & $\begin{array}{c}\text { março/ } \\
\text { abril } 2020\end{array}$ & $\begin{array}{c}\text { março } \\
2019\end{array}$ & abril 2019 & $\begin{array}{c}\text { março/abril } \\
2019\end{array}$ & var abs & $\operatorname{var} \%$ \\
\hline $\mathrm{N}^{\circ}$ Consultas Presenciais & 203 & 21 & 224 & 401 & 377 & 778 & -554 & $-71 \%$ \\
\hline No Teleconsultas & - & 30 & 30 & - & - & - & - & - \\
\hline Visitas de Especialista (AP, INT, UCIP) & 4 & 0 & 4 & 3 & 9 & 12 & -8 & $-67 \%$ \\
\hline$N^{\circ} E C G$ & 702 & 295 & 997 & 974 & 880 & 1854 & -857 & $-46 \%$ \\
\hline$N^{\circ} \mathrm{ECO}$ & 229 & 67 & 296 & 385 & 376 & 761 & -465 & $-61 \%$ \\
\hline $\mathrm{N}^{\circ}$ Holter & 37 & 8 & 45 & 79 & 83 & 162 & -117 & $-72 \%$ \\
\hline$N^{\circ}$ MAPA & 28 & 6 & 34 & 49 & 41 & 90 & -56 & $-62 \%$ \\
\hline$N^{\circ} P E$ & 70 & 5 & 75 & 144 & 114 & 258 & -183 & $-71 \%$ \\
\hline
\end{tabular}

$\mathrm{AP}=$ atendimento permanente; INT=internamento; UCIP=unidade de cuidados intensivos polivalente; ECG=eletrocardiogramas; ECO=ecocardiogramas; Holter=registos de Holter; MAPA=monitorização ambulatória da pressão arterial; PE=provas de esforço; var abs=variação absoluta; var \%=percentagem de variação.

TABELA 3. Número de consultas e exames não invasivos de cardiologia para o hospital CUF Infante Santo (HCIS) - evolução no primeiro quadrimestre de 2020.

\begin{tabular}{|c|c|c|c|c|c|c|c|c|}
\hline CARDIOLOGIA HCIS & $\begin{array}{c}\text { janeiro } \\
2020\end{array}$ & $\begin{array}{c}\text { fevereiro } \\
2020\end{array}$ & $\begin{array}{c}\text { janeiro/fev } \\
2020\end{array}$ & $\begin{array}{c}\text { março } \\
2020\end{array}$ & $\begin{array}{l}\text { abril } \\
2020\end{array}$ & $\begin{array}{c}\text { março/abril } \\
2020\end{array}$ & var abs & var $\%$ \\
\hline $\mathrm{N}^{\circ}$ Consultas Presenciais & 763 & 670 & 1433 & 337 & 48 & 385 & -1048 & $-73 \%$ \\
\hline No Teleconsultas & - & - & - & - & 79 & 79 & 79 & - \\
\hline Visitas de Especialista (AP, INT, UCIP) & 8 & 14 & 22 & 14 & 3 & 17 & -5 & $-23 \%$ \\
\hline$N^{\circ} E C G$ & 1367 & 1407 & 2774 & 651 & 320 & 971 & -1803 & $-65 \%$ \\
\hline $\mathrm{N}^{\circ} \mathrm{ECO}$ & 708 & 682 & 1390 & 337 & 48 & 385 & -1005 & $-72 \%$ \\
\hline $\mathrm{N}^{\circ}$ Holter & 143 & 132 & 275 & 59 & 6 & 65 & -210 & $-76 \%$ \\
\hline$N^{\circ}$ MAPA & 49 & 59 & 108 & 28 & 6 & 34 & -74 & $-68 \%$ \\
\hline$N^{\circ} P E$ & 251 & 234 & 485 & 103 & 0 & 103 & -382 & $-79 \%$ \\
\hline
\end{tabular}

AP=atendimento permanente; INT=internamento; UCIP=unidade de cuidados intensivos polivalente; ECG=eletrocardiogramas; ECO=ecocardiogramas; Holter=registos de Holter; MAPA=monitorização ambulatória da pressão arterial; $P E=$ provas de esforço; var abs=variação absoluta; var \%=percentagem de variação.

mes não invasivos de cardiologia para os hospitais HCIS e HCP. Os dados mostram reduções superiores a 70\% para as consultas presenciais, e superiores a $60 \%$ para a quase totalidade dos exames (com exceção dos ECGs que diminuíram 46\% no HCP).
No HCIS, manteve-se uma escala presencial de cardiologistas em regime de serviços mínimos, tendo-se verificado uma diminuição mais ligeira (12\% face a igual período de 2019) no número de visitas médicas solicitadas para doentes internados (com suspeita de ou com infeção COVID-19). 
TABELA 4. Número de consultas e exames não invasivos de cardiologia para o hospital CUF Porto (HCP) - evolução no primeiro quadrimestre de 2020.

\begin{tabular}{|c|c|c|c|c|c|c|c|c|}
\hline CARDIOLOGIA HCIS & $\begin{array}{c}\text { janeiro } \\
2020\end{array}$ & $\begin{array}{c}\text { fevereiro } \\
2020\end{array}$ & $\begin{array}{c}\text { janeiro/fev } \\
2020\end{array}$ & $\begin{array}{l}\text { março } \\
2020\end{array}$ & $\begin{array}{l}\text { abril } \\
2020\end{array}$ & $\begin{array}{c}\text { março/abril } \\
2020\end{array}$ & var abs & $\operatorname{var} \%$ \\
\hline $\mathrm{N}^{\circ}$ Consultas Presenciais & 463 & 441 & 904 & 203 & 21 & 224 & -680 & $-75 \%$ \\
\hline $\mathrm{N}^{\circ}$ Teleconsultas & - & - & - & - & 30 & 30 & 30 & - \\
\hline Visitas de Especialista (AP, INT, UCIP) & 11 & 14 & 25 & 4 & 0 & 4 & -21 & $-84 \%$ \\
\hline$N^{\circ} E C G$ & 943 & 927 & 1870 & 632 & 290 & 922 & -948 & $-51 \%$ \\
\hline$N^{\circ} E C O$ & 495 & 431 & 926 & 229 & 67 & 296 & -630 & $-68 \%$ \\
\hline $\mathrm{N}^{\circ}$ Holter & 96 & 83 & 179 & 37 & 8 & 45 & -134 & $-75 \%$ \\
\hline$N^{\circ}$ MAPA & 49 & 59 & 108 & 28 & 6 & 34 & -74 & $-69 \%$ \\
\hline$N^{0} P E$ & 159 & 146 & 305 & 70 & 5 & 75 & -230 & $-75 \%$ \\
\hline
\end{tabular}

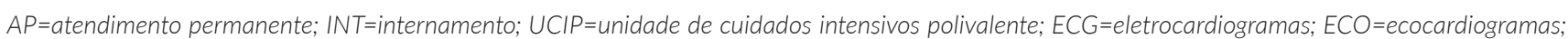
Holter=registos de Holter; MAPA=monitorização ambulatória da pressão arterial; PE=provas de esforço; var abs=variação absoluta; var \%=percentagem de variação.

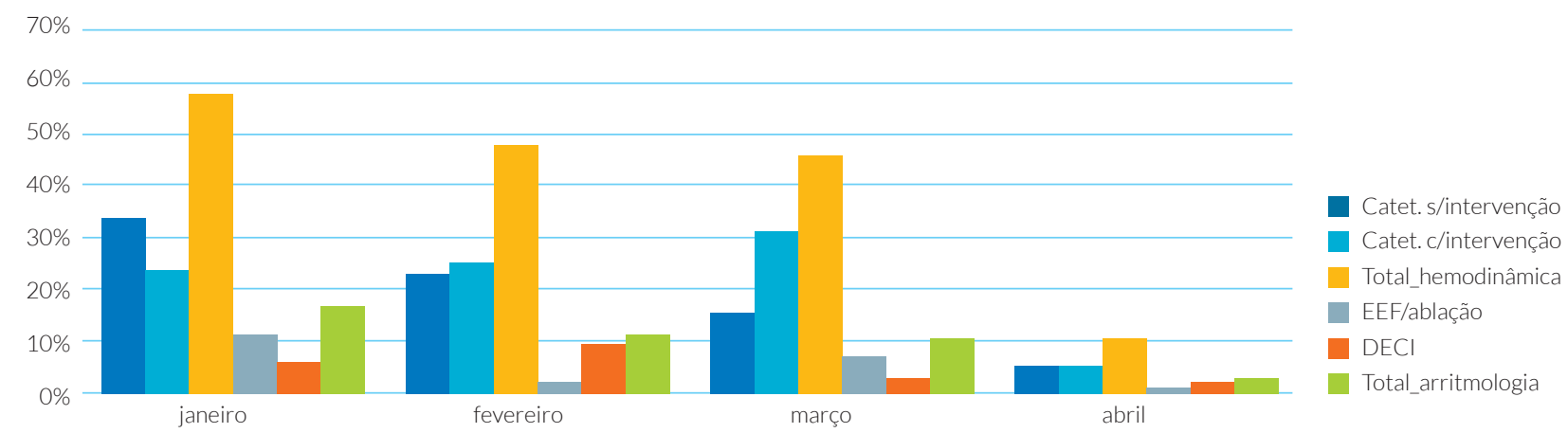

Catet=cateterismo; $E E F=e s t u d o$ eletrofisológico; $D E C l=$ dispositivos eletrónicos cardíacos implantáveis.

FIGURA 1. Evolução do número e tipo de procedimentos invasivos de cardiologia (janeiro-abril/2020) no Hospital CUF Infante Santo.

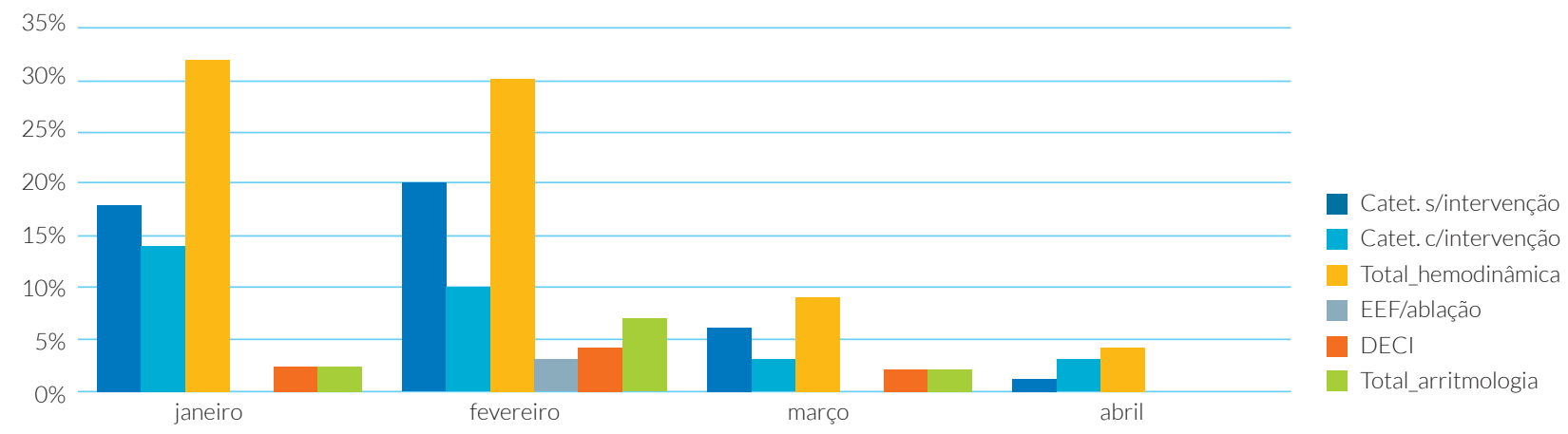

Catet=cateterismo; $E E F=e s t u d o$ eletrofisológico; $D E C l=$ dispositivos eletrónicos cardíacos implantáveis.

FIGURA 2. Evolução do número e tipo de procedimentos invasivos de cardiologia (janeiro-abril/2020) no Hospital CUF Porto.

Quando olhamos para a evolução dos números no primeiro quadrimestre de 2020 (Tabelas 3 e 4), o padrão de redução foi muito acentuado, com uma queda ainda mais marcada no mês de abril (que decorreu todo durante o período de estado de emergência), onde a atividade nestes centros de cardiologia foi direcionada somente para doentes internados e para situações urgentes ou prioritárias.
Relativamente aos procedimentos invasivos, envolvendo cateterismos cardíacos, e angioplastias e intervenção estrutural, estudos eletrofisiológicos, ablações e implantação de dispositivos eletrónicos cardíacos, as Fig.s 1 e 2 mostram a evolução dos números no período de janeiro a abril de 2020, em ambos os hospitais. Verificou-se uma redução acentuada em março e abril (ligeira no HCIS para a hemodinâmica durante o mês de 
março), com as intervenções urgentes no contexto de síndroma coronária aguda a manterem-se sem variação significativa face aos dados do mesmo período de 2019 (Fig. 3), com um total de 4 enfartes do miocárdio para cada um dos períodos no HCP, e de 16 enfartes para março e abril de 2020 versus 15 casos no período homólogo de 2019.

Estes dados confirmam uma redução muito acentuada da atividade dos Centros do Coração de dois hospitais de referência no diagnóstico e tratamento de patologias do foro da medicina cardiovascular, mostrando que, o mês de abril de 2020, que decorreu na sua totalidade em estado de emergência, apresentou uma queda ainda mais marcada que o mês de março, mercê do cancelamento de toda a atividade eletiva. Esta redução para valores mínimos, apenas compensada parcialmente pela introdução de várias dezenas de teleconsultas em abril, e pelo apoio mantido, não quantificado, a doentes e a outros especialistas através de contacto telefónico, deixa antever um aumento da procura de remarcações após o desconfinamento e retoma gradual da normalidade, com aparecimento crescente de doentes com descompensação da sua situação clínica.

Neste contexto, que se antevê difícil de gerir, há que olhar atentamente (e repensar) o modelo de funcionamento da nossa atividade hospitalar.

Além da necessidade de manter regras de segurança para doentes e profissionais de saúde, não abdicando de equipamentos de proteção adequada nem das normas de distanciamento e higienização, deveremos incentivar o recurso crescente à telemedicina e monitorização à distância, valorizando a estratificação de risco e o seguimento dos doentes, com utilização de ferramentas que permitam obter registos de variáveis como a tensão arterial, ritmo cardíaco, peso, oximetria ou atividade física, sem a necessidade de recorrer a frequentes consultas presenciais com os custos e outros fatores inerentes.

As equipas têm de estabelecer prioridades e melhorar a planificação do seu trabalho multidisciplinar com maior envolvimento e responsabilidades atribuídos a enfermeiros e cardiopneumologistas. Neste campo, o conceito de hospitalização domiciliária, permitindo cuidados assistenciais "home-based" em casos selecionados, pode vir a assumir grande utilidade, sobretudo em idosos com insuficiência cardíaca crónica.

No que se refere ao risco de infeção COVID-19, há que recuperar a confiança da população nos hospitais, procurando evitar danos cardiovasculares colaterais com atrasos nos diagnósticos e perda de tratamentos atempados. As sociedades civil e científica têm aqui um pa-
EAM - Cateterismo de urgência - HCP e HCIS

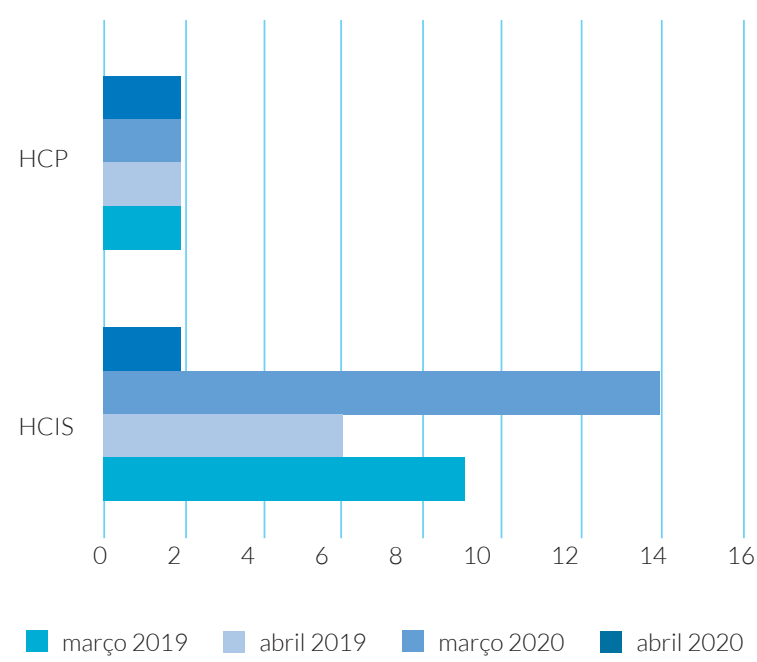

FIGURA 3. Número de casos com cateterismo de urgência e intervenção com angioplastia no Hospital CUF Porto (HCP) e no Hospital CUF Infante Santo (HCIS) (março e abril/2020 e março e abril/2019).

EAM=enfarte agudo do miocárdio.

pel de grande importância na divulgação da informação apropriada relativamente às urgências e intervenções cardiovasculares, usando os diferentes canais de comunicação.

Por outro lado, a atividade clínica em Cardiologia vai debater-se também com listas de espera, difíceis de recuperar, tendo em conta que as regras da normalização gradual implicam um número de atos médicos abaixo do habitual. Haverá, pois, pressão crescente a vários níveis sobre os profissionais de saúde, o que condicionará mais dificuldades na gestão do nosso tempo e implicará, necessariamente, bom senso nas diferentes situações com que, certamente, seremos confrontados na nossa prática diária.

Finalmente, o risco de que a acentuada redução da atividade clínica em Cardiologia durante o período da pandemia COVID-19 se possa associar a um impacto desfavorável na evolução dos doentes do foro cardiovascular, com mais complicações, morbilidade e mortalidade é real e implica a definição de estratégias concertadas para ultrapassar esse problema. Os modelos de financiamento devem contemplar a recuperação da saúde cardiovascular com projetos de telemedicina avançada, assistência domiciliária, reorganização do funcionamento das equipas hospitalares, dotando-as de mais profissionais com diferenciação em áreas que se complementem, para melhorar a dinâmica multidisciplinar na prestação das várias componentes da assistência em Cardiologia. 


\section{AGRADECIMENTOS / ACKNOWLEDGEMENTS}

Os autores agradecem a colaboração de Francisco Lourenço Mendonça (HCP), Rita Jordão e Mariana CarvaIho (HCIS) na disponibilização dos dados da atividade clínica dos centros de cardiologia.

\section{RESPONSABILIDADES ÉTICAS}

CONFLITOS DE INTERESSE: Os autores declaram a inexistência de conflitos de interesse na realização do presente trabalho.

SUPORTE FINANCEIRO: O presente trabalho não foi suportado por nenhum subsídio ou bolsa.

PROVENIÊNCIA E REVISÃO POR PARES: Não comissionado; revisão externa por pares.

\section{ETHICAL DISCLOSURES}

CONFLICTS OF INTEREST: The authors have no conflicts of interest to declare.

FINANCIAL SUPPORT: This work has not received any contribution, grant or scholarship.

PROVENANCE AND PEER REVIEW: Not commissioned; externally peer reviewed.

\section{REFERÊNCIAS}

1. Driggin E, Madhavan M, Bikdeli B, Chuich T, Laracy J, et al. Cardiovascular Considerations for Patients, Health Care Workers, and Health Systems During the Coronavirus Disease 2019 (COVID-19) Pandemic. J Am Coll Cardiol. 2020;75:2352-71. doi: 10.1016/j.jacc.2020.03.031.

2. Instituto Nacional de Estatística (INE). Lisboa: Indicadores de contexto para a pandemia COVID-19 em Portugal. [consultado maio 2020]. Available from: https://www.ine.pt/ngt server/attachfileu.jsp?look_parentBoui=427749220\&att_display=n\&att_download $=y$.

3. Maxwell YL. Cardiology After COVID-19: Physicians Predict Lasting Change. TCTMD. [accessed May 20 2020] Available from: https://www.tctmd.com/staff/yael-I-maxwell.

4. Haseltine W. An Urgent Need to Reopen Medical Care for All. ACCESS Health International. [accessed May 01 2020] Available from: https://accessh.org/press/an-urgent-need-to-reopen-medical-care-for-all/. 